\title{
Are GAPT charges really charges?
}

\author{
WE Richter ${ }^{a, b^{*}}$, LJ Duarte ${ }^{b}$, RE Bruns $^{b}$ \\ $a$ - Department of Chemical Engineering, Federal University of Technology - Paraná. Ponta Grossa-PR, Brazil. \\ $b$ - Institute of Chemistry, State University of Campinas. Campinas-SP, Brazil.
}

\section{- Abstract}

7 GAPT has turned into a very popular charge model since it was proposed three decades ago. During this s period, several works aiming to compare different partition schemes have included it among their tested models.

9 Nonetheless, GAPT exhibits a set of unique features that prevent it from being directly comparable to "standard" 10 partition schemes. We take this opportunity the explore some of these features, mainly related to the need of 11 evaluating multiple geometries and the dynamic character of GAPT, and show how to obtain the static and 12 dynamic parts of GAPT from any static charge model in the literature. We also present a conceptual evaluation 13 of charge models that aims to explain, at least partially, why GAPT and QTAIM charges are strongly correlated 14 to one another, even though they seem to be constructed under very different frameworks. Not only are they 15 the sole models whose definitions admit direct comparison between theoretical and experimental values, both are 16 deeply ingrained with the response of the electronic density to nuclear displacements.

\author{
17 Corresponding author \\ Prof. Roy Edward Bruns, emeritus

The concept of atomic charge in chemistry is ubiquitous in the same measure as it is evasive. It is promptly invoked in all kinds of discussions, from molecular properties to reaction mechanisms while at the same moment its very physical significance is debatable. This endless dispute has produced dozens of different partition schemes interpreted as atomic charges $[1,2,3,4,5,6,7,8,9,10]$ and new models continue to be proposed. Each of them has its own pros and cons which quite often involve feasibility, reproducibility, transferability, basis set dependency, geometry dependency and computational cost. Another desirable criteria is reprodution of the total electric dipole moment, even though a number of these do not fulfill this requirement. The appearance of studies 

58 correlation.

\section{s9 2 GAPT charges, IR intensities and polar tensors}

60 The GAPT charges have their origin in experimental infrared spectroscopy, specifically infrared intensities that

carrying comparisons between such models is natural, aiming to find the most suitable one to a given purpose or the one whose results are more meaningful in terms of what is expected from chemical insight[11, 12, 13, 14, 15, 16]. On the myriad of different partition schemes, Koritsanszky and Coppens point out that "the definition of net atomic charge introduces a much larger, conceptual, variation than uncertainties in either experiment or theory. At the current state of the art, differences between experiment and theory for a given molecule appear small compared with differences between alternative definitions" [17].

Among all charge definitions available in the literature, the GAPT (Generalized Atomic Polar Tensor) can be completely determined from experimental data, infrared intensities and frequencies and equilibrium dipole moments and molecular geometries. Its origin comes from the mean dipole moment derivative appearing soon after the introduction of the polar tensor formalism by Morcillo and co-workers [18] and later extended by Person and Newton [19]. Mean dipole moment derivatives calculated from experimental atomic polar tensors have been studied for both diatomic [20] and polyatomic [21] molecules. This parameter measures the average change in molecular dipole moment for atomic Cartesian displacements of each atom in the molecule. Cioslowski [8] recognized the potential use of the mean dipole moment derivative as a charge parameter owing to its advantageous mathematical properties such as relative invariance to basis set changes. Since then GAPTs have been used in hundreds of applications. It is worth mentioning that GAPT charges belong to the select group of charges accessible by both theory and experiment, and a compilation of experimentally determined GAPT charges was published just a few years ago [22].

Two recent and very interesting contributions from Cho et al. [15] and Manz [16] reported careful statistical analyses over more than twenty different schemes for computing atomic charges. Although aiming at different goals, they report similar results concerning the correlation between the various partition schemes among each other. For instance, both present a correlation matrix that is blocked around some schemes under similar approaches, e.g. the group derived from electrostatic potentials (MK, CHELPG, HLY, RESP) and the group derived from deformation densities (Hirshfeld, Voronoi). They also report a quite high correlation between GAPT, QTAIM, which surprised the authors [15]. Considering the profound differences from GAPT to any other charge model in the literature, which were not fully evaluated by these authors, and considering this somewhat surprising correlation between GAPT and QTAIM, we aim to explore some of their features that can help demistify this are proportional to the squares of the dipole moment derivatives with respect to their normal cooordinates,

$$
A_{k}=\left(\frac{N_{A} \pi}{3 c^{2}}\right)\left(\frac{\partial \vec{p}}{\partial Q_{k}}\right)^{2} \quad k=1,2, \ldots, 3 N-6
$$


62 with $N_{A}$ and $c$ representing Avogadro's constant and the speed of light [23, 24]. These derivatives can be

63 transformed into atomic Cartesian coordinates resulting in atomic polar tensors (APT's) [18, 19]:

$$
\boldsymbol{P}_{\boldsymbol{X}}^{(\boldsymbol{j})}=\left[\begin{array}{lll}
\left(\frac{\partial p_{x}}{\partial x_{j}}\right) & \left(\frac{\partial p_{x}}{\partial y_{j}}\right) & \left(\frac{\partial p_{x}}{\partial z_{j}}\right) \\
\left(\frac{\partial p_{y}}{\partial x_{j}}\right) & \left(\frac{\partial p_{y}}{\partial y_{j}}\right) & \left(\frac{\partial p_{y}}{\partial z_{j}}\right) \\
\left(\frac{\partial p_{z}}{\partial x_{j}}\right) & \left(\frac{\partial p_{z}}{\partial y_{j}}\right) & \left(\frac{\partial p_{z}}{\partial z_{j}}\right)
\end{array}\right] \quad j=1,2, \ldots, N
$$

${ }_{64}$ for an $N$ atom molecule. Although experimental gas-phase integrated intensities for all normal modes have been 65 measured for only about a hundred molecules they are accurately calculated by high quality ab initio quantum 66 mechanical methods [25, 26].

67 The mean dipole moment derivative, now popularly known as the GAPT charge, has been defined as one-third

68 of the trace of the atomic polar tensor [8],

$$
q_{j}^{G A P T}=\overline{p_{j}}=\frac{1}{3}\left[\left(\frac{\partial p_{x}}{\partial x_{j}}\right)+\left(\frac{\partial p_{y}}{\partial y_{j}}\right)+\left(\frac{\partial p_{z}}{\partial z_{j}}\right)\right]
$$

The reader should notice that it is the molecular dipole moment that is being perturbed by the displacement of the $j^{\text {th }}$ atom in Eq. (3), so all the electrons are being considered. The separation is not based on electronic atomic densities, but actually on atomic displacements along the Cartesian coordinates (for instance, check the labels on Eq. (5) of Ref. [8]). It is a criterion based on movement rather than an electronic one that defines the atomic contribution. The key point is that the GAPT charge shows how a given atom affects the molecular dipole moment assuming it is the only atom moving. The extent of this perturbation is of course primarily related to the electronic environment around the displaced atom, and more or less polarizable atoms, when displaced, will perturb the molecular dipole moment differently; however, we need to keep in mind that all electrons are, in principle, being perturbed, even those far away from the moving atom.

This is actually the main feature differing GAPT from nearly all the remaining charge models available in the literature: while they deal with static (usually equilibrium, but not restricted to) geometries, the charges obtained from them are also "static charges". In contrast, GAPT charges are "dynamic" since they take into account the dynamic nature of the vibrational motion. One must stress that in nature atoms within a molecule are never static at their equilibrium geometries; unfortunately, most of the studies comparing atomic charges from different procedures totally ignore calculations on displaced, non-equilibrium geometries. Another important difference is that the GAPT charge is the only partition scheme that cannot be evaluated based only on a single-point calculation since, by its own definition, the molecular dipole moment needs to be evaluated at multiple different geometries.

Because the GAPT charges are not static, but actually dynamic ones, it is desirable to find a way of relating 
s8 them to the static charges available in the literature. If the molecule is not planar, all partition schemes able

89 to reproduce the total dipole moment can, in principle, be used to obtain GAPT charges. Among the several

9o models that fulfill this condition, all of them belong to one of the two following categories:

$$
\vec{p}=\sum_{i=1}^{N} \vec{p}_{i} \quad \begin{cases}\vec{p}=\sum_{i=1}^{N}\left(q_{i} \cdot \vec{r}_{i}\right) & \text { (charge-only models) } \\ \vec{p}=\sum_{i=1}^{N}\left(q_{i} \cdot \vec{r}_{i}+\vec{m}_{i}\right) & \text { (charge-and-dipole models) }\end{cases}
$$

91 for which $q_{i}, \overrightarrow{r_{i}}$ and $\vec{m}_{i}$ stand for the atomic charge, position vector and intratomic dipole of the $i^{t h}$ atom,

92 respectively. It should be mentioned that these $i$ indexes are related to the atomic contributions to the electronic

93 dipole moment whereas the $j$ index used for the displacements in earlier equations. The substitution of each case

94 of Eq. (4) into Eq. (2) leads to a partition of the APT in two (if a charge-only model was used) or three (if a charge-and-dipole model is used) terms:

$$
\begin{array}{lr}
\boldsymbol{P}_{\boldsymbol{X}}^{(j)}=\boldsymbol{P}_{\boldsymbol{X}, \boldsymbol{C}}^{(j)}+\boldsymbol{P}_{\boldsymbol{X}, \boldsymbol{C T}}^{(j)} & \text { (under charge-only models) } \\
\boldsymbol{P}_{\boldsymbol{X}}^{(j)}=\boldsymbol{P}_{\boldsymbol{X}, \boldsymbol{C}}^{(j)}+\boldsymbol{P}_{\boldsymbol{X}, \boldsymbol{C T}}^{(j)}+\boldsymbol{P}_{\boldsymbol{X}, \boldsymbol{D} \boldsymbol{P}}^{(j)} & \text { (under charge-and-dipole models) }
\end{array}
$$

It is easy to reach this conclusion: if charge--only models are used, the derivatives in Eq. (2) require the application of the chain rule on the $q_{i} \cdot \overrightarrow{r_{i}}$ product, leading to two sets of derivatives, which ultimately can be expressed as two sets of complementary polar tensors, here named Charge (C) and Charge Transfer (CT). If atomic dipoles are also included, then a third set of derivatives will appears, expressed as a third polar tensor, named Dipolar Polarization (DP). In either case, the sum of the C, CT and DP (if present) polar tensors must recover the total (experimentally determined) APT. The Charge tensor is a diagonal matrix composed of the atomic charges from the equilibrium geometry, while the Charge Transfer tensor concerns the fluctuations, or rearrangements, of the electronic density that occur as a response to the atomic displacements. Similarly to the CT tensor, the DP one will concern the modifications of the intratomic dipoles that are also caused by displacements.

The immediate consequence of the latter equations is that if the APT can be separated into smaller terms, the GAPT charge, as defined from the total APT, can be divided into equivalent terms. In fact, it can be divided into C, CT and DP terms by taking one-third of each of the polar tensors in the above equations, so:

$$
\begin{aligned}
& q_{j}^{G A P T}=q_{j}^{C}+q_{j}^{C T} \quad \text { (under charge-only models) } \\
& q_{j}^{G A P T}=q_{j}^{C}+q_{j}^{C T}+q_{j}^{D P} \quad \text { (under charge-and-dipole models) }
\end{aligned}
$$
model we choose to determine them, there is no way of avoiding the charge transfers and dipolar polarizations, 
111

Table 1: Charge (C), Charge Transfer (CT) and Dipolar Polarization (DP) contributions to the total GAPT charge as obtained from QTAIM atomic charges and dipoles (all terms in units of electrons, e).

\begin{tabular}{|c|c|c|c|c|c|}
\hline Molecule & Atom & $\mathbf{C}$ & CT & DP & GAPT \\
\hline \multirow[t]{2}{*}{$\mathrm{CH}_{4}$} & C & 0.013 & -0.354 & 0.362 & 0.021 \\
\hline & $\mathbf{H}$ & -0.003 & 0.089 & -0.091 & -0.005 \\
\hline \multirow[t]{3}{*}{$\mathrm{CH}_{3} \mathrm{~F}$} & $\mathbf{C}$ & 0.650 & -0.360 & 0.289 & 0.579 \\
\hline & $\mathbf{F}$ & -0.699 & 0.145 & 0.064 & -0.490 \\
\hline & $\mathbf{H}$ & 0.016 & 0.072 & -0.119 & -0.031 \\
\hline \multirow[t]{3}{*}{$\mathrm{CH}_{2} \mathrm{~F}_{2}$} & C & 1.314 & -0.412 & 0.217 & 1.119 \\
\hline & $\mathbf{F}$ & -0.703 & 0.133 & 0.051 & -0.518 \\
\hline & $\mathbf{H}$ & 0.046 & 0.073 & -0.159 & -0.040 \\
\hline \multirow[t]{3}{*}{$\mathrm{CHF}_{3}$} & $\mathbf{C}$ & 2.021 & -0.557 & 0.135 & 1.598 \\
\hline & $\mathbf{H}$ & 0.091 & 0.101 & -0.221 & -0.029 \\
\hline & $\mathbf{F}$ & -0.704 & 0.152 & 0.029 & -0.523 \\
\hline \multirow[t]{2}{*}{$C F_{4}$} & C & 2.786 & -0.845 & 0.107 & 2.048 \\
\hline & $\mathbf{F}$ & -0.696 & 0.211 & -0.028 & -0.513 \\
\hline \multirow[t]{3}{*}{$\mathrm{CClF}_{3}$} & $\mathbf{C}$ & 2.211 & -0.202 & -0.119 & 1.890 \\
\hline & $\mathrm{Cl}$ & -0.135 & -0.199 & 0.076 & -0.258 \\
\hline & $\mathbf{F}$ & -0.692 & 0.134 & 0.018 & -0.540 \\
\hline \multirow[t]{3}{*}{$\mathrm{CCl}_{2} \mathrm{~F}_{2}$} & $\mathbf{C}$ & 1.635 & 0.350 & -0.269 & 1.717 \\
\hline & $\mathbf{F}$ & -0.687 & 0.082 & 0.046 & -0.559 \\
\hline & $\mathrm{Cl}$ & -0.131 & -0.257 & 0.093 & -0.295 \\
\hline \multirow[t]{3}{*}{$\mathrm{CCl}_{3} \mathrm{~F}$} & C & 1.052 & 0.822 & -0.398 & 1.475 \\
\hline & $\mathbf{F}$ & -0.680 & 0.056 & 0.061 & -0.564 \\
\hline & $\mathrm{Cl}$ & -0.124 & -0.292 & 0.111 & -0.305 \\
\hline \multirow[t]{2}{*}{$\mathrm{CCl}_{4}$} & C & 0.466 & 1.237 & -0.510 & 1.194 \\
\hline & $\mathrm{Cl}$ & -0.117 & -0.309 & 0.124 & -0.301 \\
\hline \multirow[t]{3}{*}{$\mathrm{CHCl3}$} & $\mathbf{C}$ & 0.363 & 0.792 & -0.234 & 0.921 \\
\hline & $\mathbf{H}$ & 0.125 & -0.055 & -0.103 & -0.034 \\
\hline & $\mathrm{Cl}$ & -0.163 & -0.246 & 0.114 & -0.295 \\
\hline \multirow[t]{3}{*}{$\mathrm{CH}_{2} \mathrm{Cl}_{2}$} & $\mathbf{C}$ & 0.255 & 0.357 & 0.002 & 0.614 \\
\hline & $\mathrm{Cl}$ & -0.214 & -0.174 & 0.099 & -0.289 \\
\hline & $\mathbf{H}$ & 0.087 & -0.004 & -0.101 & -0.019 \\
\hline \multirow[t]{3}{*}{$\mathrm{CH}_{3} \mathrm{Cl}$} & $\mathbf{C}$ & 0.140 & -0.039 & 0.198 & 0.299 \\
\hline & $\mathrm{Cl}$ & -0.271 & -0.092 & 0.087 & -0.276 \\
\hline & $\mathbf{H}$ & 0.044 & 0.044 & -0.095 & -0.008 \\
\hline
\end{tabular}


Table 2: Charge (C), Charge Transfer (CT) and Dipolar Polarization (DP) contributions to the total GAPT charge as obtained from Hirshfeld atomic charges and dipoles (all terms in units of electrons, e).

\begin{tabular}{|c|c|c|c|c|c|}
\hline Molecule & Atom & $\mathrm{C}$ & CT & DP & GAPT \\
\hline \multirow{2}{*}{$\mathrm{CH}_{4}$} & C & -0.112 & 0.036 & 0.097 & 0.021 \\
\hline & $\mathbf{H}$ & 0.028 & -0.009 & -0.024 & -0.005 \\
\hline \multirow[t]{3}{*}{$\mathrm{CH}_{3} \mathrm{~F}$} & $\mathbf{C}$ & 0.051 & 0.294 & 0.235 & 0.579 \\
\hline & $\mathbf{F}$ & -0.160 & -0.201 & -0.125 & -0.486 \\
\hline & $\mathbf{H}$ & 0.037 & -0.031 & -0.037 & -0.031 \\
\hline \multirow[t]{3}{*}{$\mathrm{CH}_{2} \mathrm{~F}_{2}$} & $\mathbf{C}$ & 0.185 & 0.591 & 0.341 & 1.117 \\
\hline & $\mathbf{F}$ & -0.137 & -0.253 & -0.127 & -0.517 \\
\hline & $\mathbf{H}$ & 0.044 & -0.043 & -0.043 & -0.042 \\
\hline \multirow[t]{3}{*}{$\mathrm{CHF}_{3}$} & $\mathbf{C}$ & 0.300 & 0.906 & 0.397 & 1.604 \\
\hline & $\mathbf{H}$ & 0.055 & -0.041 & -0.045 & -0.030 \\
\hline & $\mathbf{F}$ & -0.119 & -0.288 & -0.117 & -0.524 \\
\hline \multirow{2}{*}{$C F_{4}$} & C & 0.410 & 1.230 & 0.415 & 2.055 \\
\hline & $\mathbf{F}$ & -0.103 & -0.307 & -0.104 & -0.514 \\
\hline \multirow[t]{3}{*}{$\mathrm{CClF}_{3}$} & $\mathbf{C}$ & 0.355 & 1.300 & 0.251 & 1.906 \\
\hline & $\mathrm{Cl}$ & -0.058 & -0.221 & 0.014 & -0.265 \\
\hline & $\mathbf{F}$ & -0.099 & -0.360 & -0.088 & -0.547 \\
\hline \multirow[t]{3}{*}{$C C l_{2} F_{2}$} & $\mathrm{C}$ & 0.301 & 1.311 & 0.105 & 1.717 \\
\hline & $\mathbf{F}$ & -0.094 & -0.397 & -0.072 & -0.563 \\
\hline & $\mathrm{Cl}$ & -0.056 & -0.258 & 0.019 & -0.295 \\
\hline \multirow[t]{3}{*}{$C C l_{3} F$} & $\mathbf{C}$ & 0.248 & 1.259 & -0.023 & 1.484 \\
\hline & $\mathbf{F}$ & -0.091 & -0.422 & -0.053 & -0.566 \\
\hline & $\mathrm{Cl}$ & -0.052 & -0.279 & 0.026 & -0.306 \\
\hline \multirow[t]{2}{*}{$\mathrm{CCl}_{4}$} & $\mathrm{C}$ & 0.194 & 1.147 & -0.129 & 1.212 \\
\hline & $\mathrm{Cl}$ & -0.048 & -0.287 & 0.032 & -0.303 \\
\hline \multirow[t]{3}{*}{$\mathrm{CHCl}_{3}$} & $\mathbf{C}$ & 0.135 & 0.813 & -0.023 & 0.925 \\
\hline & $\mathbf{H}$ & 0.059 & -0.060 & -0.036 & -0.038 \\
\hline & $\mathrm{Cl}$ & -0.065 & -0.251 & 0.020 & -0.296 \\
\hline \multirow[t]{3}{*}{$\mathrm{CH}_{2} \mathrm{Cl}_{2}$} & $\mathbf{C}$ & 0.073 & 0.490 & 0.051 & 0.614 \\
\hline & $\mathrm{Cl}$ & -0.090 & -0.205 & 0.006 & -0.289 \\
\hline & $\mathbf{H}$ & 0.054 & -0.040 & -0.032 & -0.018 \\
\hline \multirow[t]{3}{*}{$\mathrm{CH}_{3} \mathrm{Cl}$} & $\mathbf{C}$ & -0.005 & 0.215 & 0.091 & 0.301 \\
\hline & $\mathrm{Cl}$ & -0.128 & -0.140 & -0.007 & -0.276 \\
\hline & $\mathbf{H}$ & 0.044 & -0.025 & -0.028 & -0.008 \\
\hline
\end{tabular}


Table 3: Charge (C), Charge Transfer (CT) and Dipolar Polarization (DP) contributions to the total GAPT charge as obtained from CHELPG atomic charges and dipoles (all terms in units of electrons, e).

\begin{tabular}{|c|c|c|c|c|c|}
\hline Molecule & Atom & $\mathrm{C}$ & CT & DP & GAPT \\
\hline \multirow[t]{2}{*}{$\mathrm{CH}_{4}$} & $\mathbf{C}$ & 1.050 & 0.555 & -1.584 & 0.021 \\
\hline & $\mathbf{H}$ & -0.263 & -0.134 & 0.392 & -0.005 \\
\hline \multirow[t]{3}{*}{$\mathrm{CH}_{3} \mathrm{~F}$} & $\mathbf{C}$ & 1.596 & 0.471 & -1.488 & 0.579 \\
\hline & $\mathbf{F}$ & -0.471 & -0.346 & 0.328 & -0.489 \\
\hline & $\mathbf{H}$ & -0.375 & 0.054 & 0.289 & -0.032 \\
\hline \multirow[t]{3}{*}{$\mathrm{CH}_{2} \mathrm{~F}_{2}$} & $\mathrm{C}$ & 1.759 & 0.560 & -1.202 & 1.117 \\
\hline & $\mathbf{F}$ & -0.559 & -0.224 & 0.264 & -0.520 \\
\hline & $\mathbf{H}$ & -0.320 & -0.058 & 0.338 & -0.040 \\
\hline \multirow[t]{3}{*}{$\mathrm{CHF}_{3}$} & $\mathrm{C}$ & 1.996 & 0.747 & -1.140 & 1.604 \\
\hline & $\mathbf{H}$ & -0.288 & -0.054 & 0.314 & -0.028 \\
\hline & $\mathbf{F}$ & -0.570 & -0.241 & 0.287 & -0.524 \\
\hline \multirow[t]{2}{*}{$C F_{4}$} & C & 2.341 & 1.019 & -1.305 & 2.055 \\
\hline & $\mathbf{F}$ & -0.585 & -0.271 & 0.342 & -0.515 \\
\hline \multirow[t]{3}{*}{$\mathrm{CClF}_{3}$} & $\mathbf{C}$ & 2.499 & 1.060 & -1.653 & 1.906 \\
\hline & $\mathrm{Cl}$ & -0.594 & -0.041 & 0.369 & -0.266 \\
\hline & $\mathbf{F}$ & -0.635 & -0.411 & 0.501 & -0.546 \\
\hline \multirow[t]{3}{*}{$\mathrm{CCl}_{2} \mathrm{~F}_{2}$} & $\mathbf{C}$ & 2.694 & 0.989 & -1.965 & 1.718 \\
\hline & $\mathbf{F}$ & -0.705 & -0.395 & 0.541 & -0.559 \\
\hline & $\mathrm{Cl}$ & -0.642 & -0.077 & 0.424 & -0.295 \\
\hline \multirow[t]{3}{*}{$\mathrm{CCl}_{3} \mathrm{~F}$} & $\mathbf{C}$ & 2.731 & 0.880 & -2.128 & 1.484 \\
\hline & $\mathbf{F}$ & -0.721 & -0.846 & 1.009 & -0.558 \\
\hline & $\mathrm{Cl}$ & -0.670 & 0.328 & 0.040 & -0.302 \\
\hline \multirow[t]{2}{*}{$\mathrm{CCl}_{4}$} & $\mathbf{C}$ & 2.904 & 0.658 & -2.350 & 1.212 \\
\hline & $\mathrm{Cl}$ & -0.726 & -0.208 & 0.630 & -0.304 \\
\hline \multirow[t]{3}{*}{$\mathrm{CHCl}_{3}$} & $\mathbf{C}$ & 2.735 & 0.408 & -2.218 & 0.925 \\
\hline & $\mathbf{H}$ & -0.664 & 0.325 & 0.303 & -0.035 \\
\hline & $\mathrm{Cl}$ & -0.690 & -0.213 & 0.609 & -0.294 \\
\hline \multirow[t]{3}{*}{$\mathrm{CH}_{2} \mathrm{Cl}_{2}$} & $\mathbf{C}$ & 2.481 & 0.339 & -2.206 & 0.614 \\
\hline & $\mathrm{Cl}$ & -0.670 & -0.367 & 0.747 & -0.290 \\
\hline & $\mathbf{H}$ & -0.571 & -0.063 & 0.614 & -0.020 \\
\hline \multirow[t]{3}{*}{$\mathrm{CH}_{3} \mathrm{Cl}$} & $\mathbf{C}$ & 2.072 & 0.408 & -2.180 & 0.301 \\
\hline & $\mathrm{Cl}$ & -0.620 & 0.026 & 0.317 & -0.277 \\
\hline & $\mathbf{H}$ & -0.484 & -0.191 & 0.666 & -0.009 \\
\hline
\end{tabular}


Table 4: Charge (C) and Charge Transfer (CT) contributions to the total GAPT charge as obtained from CHELPG atomic charges [no dipoles] (all terms in units of electrons, e) .

\begin{tabular}{|c|c|c|c|c|c|}
\hline Molecule & Atom & $\mathbf{C}$ & CT & DP & GAPT \\
\hline \multirow[t]{2}{*}{$\mathrm{CH}_{4}$} & $\mathrm{C}$ & -0.360 & 0.381 & 0.000 & 0.021 \\
\hline & $\mathbf{H}$ & 0.090 & -0.095 & 0.000 & -0.005 \\
\hline \multirow[t]{3}{*}{$\mathrm{CH}_{3} \mathrm{~F}$} & $\mathrm{C}$ & 0.144 & 0.435 & 0.000 & 0.579 \\
\hline & $\mathbf{F}$ & -0.247 & -0.239 & 0.000 & -0.487 \\
\hline & $\mathbf{H}$ & 0.035 & -0.065 & 0.000 & -0.031 \\
\hline \multirow[t]{3}{*}{$\mathrm{CH}_{2} \mathrm{~F}_{2}$} & $\mathbf{C}$ & 0.389 & 0.728 & 0.000 & 1.117 \\
\hline & $\mathbf{F}$ & -0.227 & -0.291 & 0.000 & -0.518 \\
\hline & $\mathbf{H}$ & 0.032 & -0.074 & 0.000 & -0.042 \\
\hline \multirow[t]{3}{*}{$\mathrm{CHF}_{3}$} & $\mathrm{C}$ & 0.540 & 1.064 & 0.000 & 1.604 \\
\hline & $\mathbf{H}$ & 0.052 & -0.082 & 0.000 & -0.030 \\
\hline & $\mathbf{F}$ & -0.197 & -0.327 & 0.000 & -0.524 \\
\hline \multirow[t]{2}{*}{$C F_{4}$} & $\mathrm{C}$ & 0.682 & 1.373 & 0.000 & 2.055 \\
\hline & $\mathbf{F}$ & -0.170 & -0.343 & 0.000 & -0.514 \\
\hline \multirow[t]{3}{*}{$\mathrm{CClF}_{3}$} & $\mathbf{C}$ & 0.334 & 1.572 & 0.000 & 1.906 \\
\hline & $\mathrm{Cl}$ & -0.033 & -0.232 & 0.000 & -0.265 \\
\hline & $\mathbf{F}$ & -0.100 & -0.447 & 0.000 & -0.547 \\
\hline \multirow[t]{3}{*}{$C C l_{2} F_{2}$} & $\mathbf{C}$ & 0.072 & 1.646 & 0.000 & 1.717 \\
\hline & $\mathbf{F}$ & -0.045 & -0.517 & 0.000 & -0.562 \\
\hline & $\mathrm{Cl}$ & 0.009 & -0.303 & 0.000 & -0.294 \\
\hline \multirow[t]{3}{*}{$\mathrm{CCl}_{3} \mathrm{~F}$} & $\mathbf{C}$ & -0.088 & 1.571 & 0.000 & 1.484 \\
\hline & $\mathbf{F}$ & -0.016 & -0.550 & 0.000 & -0.566 \\
\hline & $\mathrm{Cl}$ & 0.035 & -0.341 & 0.000 & -0.306 \\
\hline \multirow[t]{2}{*}{$\mathrm{CCl}_{4}$} & $\mathrm{C}$ & -0.270 & 1.481 & 0.000 & 1.212 \\
\hline & $\mathrm{Cl}$ & 0.067 & -0.370 & 0.000 & -0.303 \\
\hline \multirow[t]{3}{*}{$\mathrm{CHCl}_{3}$} & $\mathrm{C}$ & -0.135 & 1.059 & 0.000 & 0.925 \\
\hline & $\mathbf{H}$ & 0.186 & -0.224 & 0.000 & -0.038 \\
\hline & $\mathrm{Cl}$ & -0.017 & -0.280 & 0.000 & -0.297 \\
\hline \multirow[t]{3}{*}{$\mathrm{CH}_{2} \mathrm{Cl}_{2}$} & $\mathrm{C}$ & -0.111 & 0.725 & 0.000 & 0.614 \\
\hline & $\mathrm{Cl}$ & -0.087 & -0.203 & 0.000 & -0.290 \\
\hline & $\mathbf{H}$ & 0.143 & -0.162 & 0.000 & -0.019 \\
\hline \multirow[t]{3}{*}{$\mathrm{CH}_{3} \mathrm{Cl}$} & $\mathrm{C}$ & -0.157 & 0.458 & 0.000 & 0.301 \\
\hline & $\mathrm{Cl}$ & -0.161 & -0.114 & 0.000 & -0.276 \\
\hline & $\mathbf{H}$ & 0.106 & -0.115 & 0.000 & -0.008 \\
\hline
\end{tabular}

Inspection of Tables 1-4 confirms that the total GAPT charge values are absolutely equal within numerical accuracy. This is not a surprise as among these four partition schemes, two of them naturally reproduce the total dipole moment and the remaining two were constrained to do so; in other words, the intensities and polar tensors will be calculated to be the same within numerical error. On the other hand, it is easily seen that the individual C, CT and DP terms (only C,CT for CHELPG-q) are indeed very different for each model. These differences are better visualized in Figure 1.

Can be see there the four models show stricking differences for the C, CT and DP parcels in all cases. First analyzing the GAPT for carbon in methane $(+0.021 e)$, GAPT obtained using QTAIM parameters shows that the equilibrium (static) charge is nearly zero, and the CT and DP components are larger but have opposite signs. This is expected from chemical insight because the IR intensities in hydrocarbons are expected to be described almost solely from dynamic terms, as in thees molecules the atomic charges are expected to be nearly zero [27]. Hirshfeld static charge is still small, though a bit larger than QTAIM's in magnitude, but the most important difference is that now the CT and DP terms no longer have opposite signs, but reiforce one another. One can 

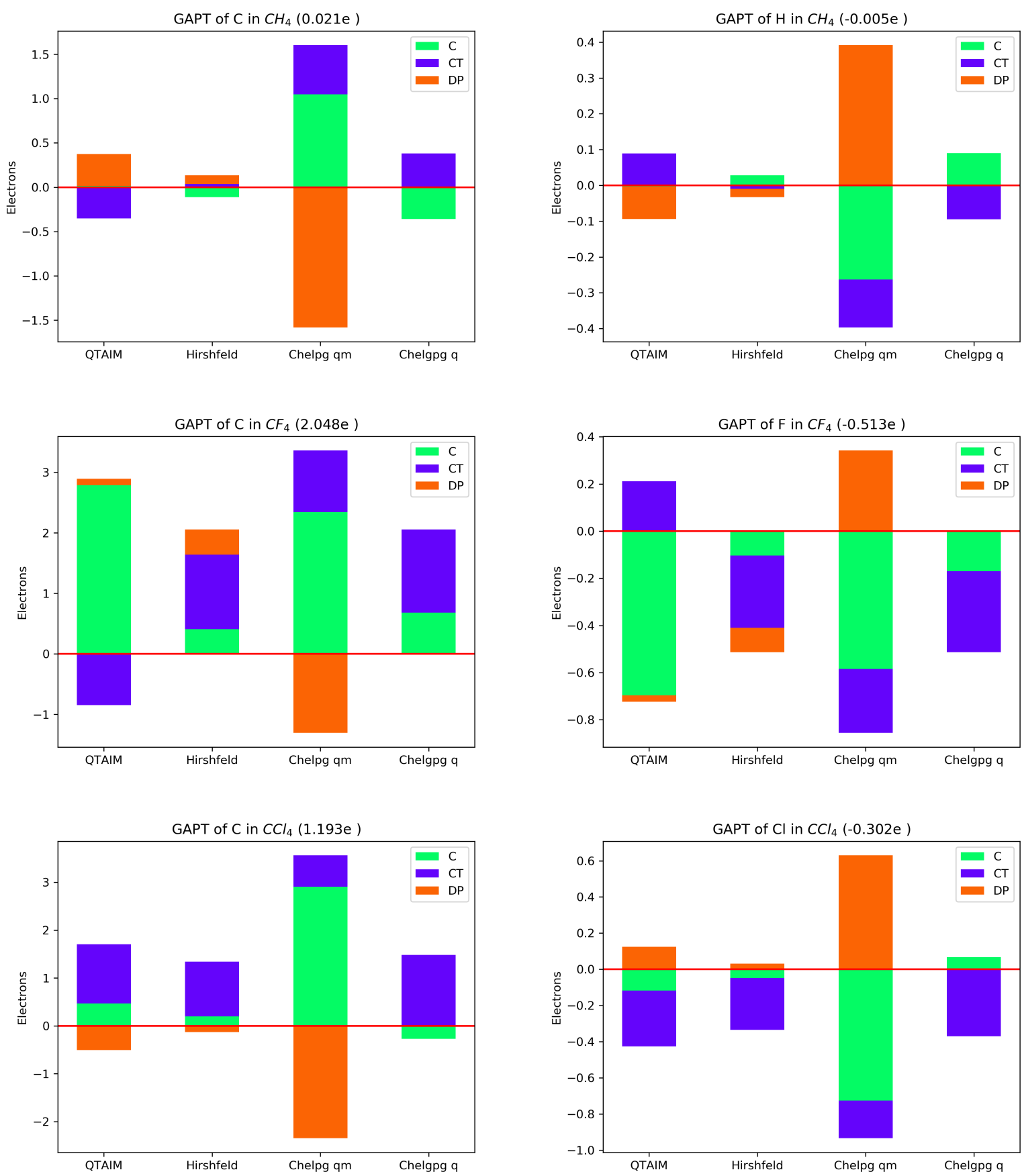

Figure 1: C, CT and DP contributions from QTAIM, Hirshfeld, CHELPG with charges-and-dipoles (CHELPG$\mathrm{qm}$ ) and CHELPG with only point-charges (CHELPG-q) to GAPT charges for carbon, hydrogen, fluorine and chlorine in $C X_{4}$ molecules. All quantities in electrons. 
see that the interpretations of the GAPT charge by the different models are not necessarily equivalent to each other. The same conceptual picture could be drawn for the hydrogen results from QTAIM and Hirshfeld as well.

The CHELPG results for methane deserve a more careful evaluation. As the same total GAPT charge must be reproduced by all models, the $\mathrm{C}$ and $\mathrm{CT}$ terms for CHELPG-q combined have only a single degree of freedom. The charge term is determined directly from the equilibrium, static charges, so the CT must be such that, summed to $\mathrm{C}$, it will reproduce the total GAPT charge. As the methane GAPT charges are almost zero the $\mathrm{C}$ and CT terms from CHELPG-q must necessarily cancel each other almost perfectly, which is indeed observed. By adding an additional degree of freedom (the intratomic dipoles from CHELPG-qm), one could expect a much better description for these atoms, but this is not the case. In fact, CHELPG-qm indicates that the static atomic charge for carbon is greater than $1.00 e$ and for hydrogen is lower, i.e. more negative, than $-0.2 e$, clearly contrary to the archetype of a non-polar molecule.

CHELPG-qm interpretations are even stranger for the polar $C F_{4}$ and $C C l_{4}$ molecules. First notice that static charges from $\mathrm{CHELPG-qm}$ are larger for carbon in $C C l_{4}$ than in $C F_{4}$, opposite expectations based on electronegativity order. The same problems occurs for the terminal atoms, with chlorine (in $C C l_{4}$ ) being described as more negatively charged than fluorine (in $C F_{4}$ ). CHELPG- $\mathrm{q}$, on the other hand, shows a slightly negative carbon and a slightly positive chlorine, once more contrary to electronegativity trends. CHELPG-qm also show problems when handling $\mathrm{CHCl}_{3}$ and $\mathrm{CH}_{2} \mathrm{Cl}_{2}$, resulting in quite similar static charges for hydrogen and chlorine.

For polar molecules, QTAIM and Hirshfeld result in more reasonable interpretations. First of all, they both follow electronegativity expectations for static charges, and show a larger dynamic character for $C C l_{4}$ than for $\mathrm{CF}_{4}$, which nicely agrees with their relative polarizabilities. However, QTAIM and Hirshfeld are by no means equivalent to each other as Hirshfeld result in all $\mathrm{C}, \mathrm{CT}$ and $\mathrm{DP}$ terms having the same sign in $C F_{4}$ (positive for the carbon atom, negative for fluorine), whereas QTAIM has CT and DP terms with opposite signs for each atom, cancelling one another. QTAIM also has a greater DP term for the atoms in $C C l_{4}$, which seems to agree with the smaller positive charge for carbon and the greater polarizability of chlorine, but Hirshfeld stands the opposite: fluorine having larger intratomic dipole changes than chlorine.

Similar analyses could be carried out for all the molecules in Tables 1-4 and using other alternative partition schemes that reproduce the dipole moment derivatives; this is not the aim here, however. One can expect that each charge model will reult in different interpretations for the static and dynamic parts of the GAPT charge. It is intrinsecally dynamic, but how dynamic depends on the partition scheme chosen.

\section{The hidden correlation between QTAIM and GAPT}

We have seen that any partition scheme giving atomic charges (or charges and dipoles) which satisfactorily reproduces the total molecular dipole moment can be used to obtain partitioned GAPT charges with the same total value. However, both Cho et al. [15] and Manz [16] reported quite high (and somewhat unexpected [15]) correlations between QTAIM and GAPT which is not observed for them with any other of the more than twenty charge models investigated. We shall discuss this correlation now. 
The first similarity shared by QTAIM and GAPT and by no other model concerns observability; these are the only two models for which the input data required makes no distinction between theory or experiment. GAPT is defined in such a way that requires either an experimental of theoretical Atomic Polar Tensor as input for computing the GAPT charge. The same is true for QTAIM: it requires the molecular electron density, but the theorems therein make no distinction between experimental or theoretical densities. This feature links QTAIM and GAPT to one another and distinguishes them from any other model. The concept of atomic charge as being "experimentally observable" is at the core of a long term discussion in the literature (see, for example, the discussion in Refs. $[28,29,30]$ and references therein), but this is not the point here. We state that within each definition, QTAIM and GAPT are the only models that can have both theoretical and experimental values. Moreover, while several partition schemes are designed to satisfy specific goals (to reproduce the electrostatic potential, or to reproduce the dipole moment, etc.), QTAIM and GAPT are consequences of simple criteria (the division of the molecular density into disjoint spaces separated by zero-flux surfaces or the response of the molecular dipole to an individual atomic displacement). No grid, no fitting, no iterations, no parametrization, no database.

We have stated earlier in this text that "unfortunately, most of the studies comparing atomic charges from different procedures totally ignore calculations on displaced, non-equilibrium geometries". We demonstrated how important this is for painting the correct picture given by GAPT. About that, Bader and co-workers [31] stated that "the dipole moment is given by a sum over the net charge and first moment of every atom in a molecule. The first term leads to a charge transfer contribution $p_{c}$, the second to an atomic polarization contribution $p_{a}$. It is shown that both terms are, in general, of equal importance in determining both the static molecular dipole moment and the moment induced by a nuclear displacement. Models which employ only point charges and corresponding bond moments which follow rigidly the nuclear framework, i.e., models which approximate $p_{c}$ and ignore $p_{a}$ are shown to lead to results that are incompatible with the changes that are found to occur in a molecular charge distribution during a nuclear vibration." Further ahead they are even more incisive: "Any use of the spherical atom-charge transfer model in the description of changes in dipole moment accompanying nuclear vibrations, even when modified to include changes in the atomic charges, the so-called atomic fluxes, is still less acceptable as it cannot adequately describe the relaxations in the charge density induced by the nuclear motions."

Even though QTAIM was not designed to reproduce the changes in the dipole moments during nuclear vibrations, it satisfies this condition. This was a concern of Bader and co-workers that was fully confirmed by us when we demonstrated that coherent infrared intensity modelling can only be reached by including atomic polarizations [32]. We just demonstrated that the inclusion of atomic dipoles is a necessary but not sufficient condition to achieve an accurate description of the system; the complete failure of CHELPG (either with or without dipoles) in the aforementioned cases is an example. Moreover, two models employing atomic dipoles do not necessarily give equivalent descriptions of the system. QTAIM and Hirshfeld, for example, show a fundamental difference from one another: while for QTAIM the dipole vector caused by charge transfers is in general in the opposite direction of the dipole vector caused by the intratomic dipoles, for Hirshfeld changes in these two are alligned. This is the main reason why QTAIM charges are often considered too high and Hirshfeld charges too 
low; the QTAIM atomic dipoles trend to compensate for highy charge values, whereas for Hirshfeld they trend to compensate their low values (notice, for example, the signs for QTAIM and Hirshfeld for $C F_{4}$ in Figure 1).

An additional argument come from X-ray photoelectron spectroscopy (XPS). It is well known that coreionization energies are related to the charge distribution within the molecule and, therefore, to the atomic charges on the atoms. Moreover, since the ionization actually removes an electron from the molecule, it is obvious that the remaining electronic density will be rearranged in response to the changes in electrostatic attractions (to the nuclei) and repulsions (among the electrons). As stated by Saethre et al. [33], "one must find a way to correct for the relaxation energy before using core-ionization energies as a probe for the charge distribution." Aiming to solve this question, an important conclusion of their work is that a point-charge models do not provide a realistic description of the charge distribution away from the atom of interest, and a multipole expansion is required.

Shortly after Saethre et al., Guadagnini et al. [34] found an almost perfect correlation between 1s core-electron energies and the GAPT charges for carbon atoms in a series of halomethanes, ethanes, ethene, methylacethylene and cyclopropane. For $s p^{3}, s p^{2}$ and $s p$ carbons, respectively, the correlations were 99.6, 99.4 and 99.5\%, and if only the halomethanes were considered, a correlation of $99.96 \%$ is found. It is important to stress that only experimental data were used for both the GAPT charges and 1s core-electron energies.

Bearing all this in mind, one may interpret these findings as follows: GAPT depends on the dynamics of the electron density responding to the vibrational motion. XPS data depends on the dynamics of the electron density responding to the core-ionization. Both are intrinsic dynamic features and Saethre et al. have demonstrated the XPS data cannot be correctly assembled if a point-charge-only model is considered. QTAIM satisfies the condition for higher multipoles that are necessary to correctly describe the rearrangement of the electronic density and also correctly describe the dynamics enclosed within the GAPT charge. The correlation between GAPT and XPS is almost perfect because the GAPT charge is inherently corrected for electron density dynamics. Of the alternative ways of calculating GAPT by means of static density and its fluctuations, QTAIM and its multipoles seems to be most suitable [33].

\section{Conclusions}

The arguments herein presented are not intended to be proofs of the superiority of QTAIM compared to other partition schemes in the literature. We just aimed to shed some light into this aparently surprising correlation between GAPT and QTAIM, which seems to be, at least in part, covered by conceptual similarities within their definitions, and by the fact that the dynamics of the electronic density embraced in GAPT is better accounted for by QTAIM than by the majority of remaining partition schemes. As stated (and except for the case of planar molecules), any charge model which reproduces the dipole moment will be able to provide theoretical GAPT charge values appropriate to be compared with experimental values, but since point-charge models are inherently poor (in view of the limited amount of electronic information they deliver) and since the inclusion $a$ posteriori of intratomic dipoles (like in CHELPG-qm) shows no obvious improvement, we conclude that, when proposing and discussing atomic charge values, a solid conceptual basis is as important as (or even more than) 
the numerical results. Moreover, we feel that discussions concerning charge models would be way more fruitful if atomic dipoles were also included in the analysis rather than only atomic charges. Atomic dipoles seems a source of information as rich as the charges themselves.

\section{Acknowledgements}

WER thanks Fundação Araucária de Apoio ao Desenvolvimento Científico e Tecnológico (FAADCT/PR) for a research fellowship (89-2019/FA) and finantial support to computational facilities. LJD thanks Fundação de Amparo à Pesquisa do Estado de São Paulo (FAPESP) for a BEPE doctoral fellowship (2018/24844-7). REB acknowledges FAPESP for funding research grant (2018/08861-9) and Conselho Nacional de Desenvolvimento Científico e Tecnológico (CNPq) for a research fellowship (302574/2019-0).

\section{Data and software availability}

All fluorochloromethanes had their geometries optimized using Gaussian09 (rev. B.01) [35] at the QCISD/aug-cc-pVTZ level. G09 also delivered the full vibrational analysis, Hessian matrix and raw GAPT charges at the same level of theory. The protocol, however, is not restricted to Gaussian since these tasks can be performed by a number of codes, some of them open-source. The C, CT and DP components from GAPT were calculated by Placzek program using the equilibrium and additional $6 \mathrm{~N}$ (for an $N$-atom molecule) distorted geometries $( \pm 0.01 \AA$ on each Cartesian direction) using a well known protocol described in detail elsewhere [36, 37]. Hirshfeld atomic charges and dipoles as well CHELPG charges were obtained by Gaussian's default routines, with additional constraint for CHELPG to reproduce the correct molecular dipole moment given by the wavefunction (pop=hirshfeld,chelpg,dipole); this constraint applies only to CHELPG as Hirshfeld charges and atomic dipoles combined will naturally reproduce the dipole moment. CHELPG charges with additional intratomic dipoles were obtained by means of the (pop=chelpg,dipole,atomdip) setup. QTAIM charges and dipoles were calculated by AIMAll [38]. The Atomic Polar Tensors and also their C, CT and DP inner tensors are then calculated by Placzek using two-point numerical derivatives [36, 37]:

$$
\frac{\partial q_{i}}{\partial x_{j}}=\frac{q_{i}^{(+)}-q_{i}^{(-)}}{2 \Delta x} \quad \text { and } \quad \frac{\partial m_{i, x}}{\partial x_{j}}=\frac{m_{i, x}^{(+)}-m_{i, x}^{(-)}}{2 \Delta x}
$$

for which the $(+)$ and $(-)$ superscripts concern the positive and negative displaced geometries within a given Cartesian direction, and similar relations hold for $y$ and $z$ components. All input and output files are made available in Supplementary Information. Placzek's main output, PLACZEK.OUT, contains the C, CT, DP and (TOTAL $=\mathrm{C}+\mathrm{CT}+\mathrm{DP}$ ) Polar Tensor ready to be interpreted, as well as total and partitioned GAPT charges. Placzek program is distributed by its developer free of charge, and a copy is presented on Supplementary Information; please contact the author (WER) for further details.

These partition schemes were chosen following the "charge trio" found by Cho et al. [15] to explain the greater part of three main principal components they found: QTAIM, MBSMulliken (or NPA) and HLY. As neither NPA nor MBS-Mulliken do reproduce the total dipole moment, they were substituted by Hirshfeld, which seems to be as different from QTAIM as possible [16]. Moreover, we replaced HLY by CHELPG since both are derived from electrostatic potentials, but while HLY has not been used before in publications on this topic, CHELPG has appeared in a number of them in the last decade [36, 39, 40].

\section{References}

[1] R.S. Mulliken. Electronic population analysis on lcao-mo molecular wave functions. i. The Journal of Chemical Physics, $23: 1833,1955$.

[2] A.E. Reed, R.B. Weinstock, and F. Weinhold. Natural population analysis. The Journal of Chemical Physics, 83:735, 1985.

[3] C.M. Breneman and K.B. Wiberg. Determining atom-centered monopoles from molecular electrostatic potentials. the need for high sampling density in formamide conformational analysis. Journal of Computational Chemistry, 11:361, 1990.

[4] F.L. Hirshfeld. Bonded-atom fragments for describing molecular charge densities. Theoretica Chimica Acta, 44:129, 1977.

[5] P. Bultinck, C.V. Alsenoy, P.W. Ayers, and R. Carbo-Dorca. Critical analysis and extension of the hirshfeld atoms in molecules. The Journal of Chemical Physics, 126:144111, 2007. 
[6] R.F.W. Bader. Atoms in Molecules: a quantum theory. Clarendon Press, Oxford, 1994.

[7] C.F. Guerra, J.-W. Handgraaf, E.J. Baerends, and F.M. Bickelhaupt. Voronoi deformation density (vdd) charges: Assessment of the mulliken, bader, hirshfeld, weinhold, and vdd methods for charge analysis. Journal of Computational Chemistry, 25:189, 2004.

[8] J. Cioslowski. A new population analysis based on atomic polar tensors. Journal of the American Chemical Society, 111:8333, 1989.

[9] J.A. Montgomery, M.J. Frisch, J.W. Ochterski, and G.A. Petersson. A complete basis set model chemistry. vii. use of the minimum population localization method. The Journal of Chemical Physics, 112:6532, 2000.

[10] N.G. Limas and T.A. Manz. Introducing ddec6 atomic population analysis: part 1. charge partitioning theory and methodology. RSC Advances, 6:47771-47801, 2016.

[11] J. Meister and W.H.E. Schwarz. Principal components of ionicity. The Journal of Physical Chemistry, 98:8245, 1994.

[12] K.B. Wiberg and P.R. Rablen. Comparison of atomic charges derived via different procedures. Journal of Computational Chemistry, 14:1504-1518, 1993.

[13] K.B. Wiberg and P.R. Rablen. Atomic charges. Journal of Organic Chemistry, 83:15463-15469, 2018.

[14] V. Nikolova, D. Cheshmedzhieva, S. Ilieva, and B. Galabov. Atomic charges in describing properties of aromatic molecules. Journal of Organic Chemistry, 84:1908-1915, 2019.

[15] M. Cho, N. Sylvetsky, S. Eshafi, G. Santra, I. Efremenko, and J.M.L. Martin. The atomic partial charges arboretum: Trying to see the forest for the trees. ChemPhys Chem, 21(8):688-696, 2020.

[16] T.A. Manz. Seven confluence principles: a case study of standardized statistical analysis for 26 methods that assign net atomic charges in molecules. RSC Advances, 10:44121-44148, 2020.

[17] T.S. Koritsanszky and P. Coppens. Chemical applications of x-ray charge-density analysis. Chemical Reviews, 101:1583-1627, 2001.

[18] J.F. Biarge, J. Herranz, and J. Morcillo. Anales de la Real Sociedad Espanola de Fisica e Quimica, 57:81, 1961.

[19] W.B. Person and J.H. Newton. Dipole moment derivatives and infrared intensities. i. polar tensors. The Journal of Chemical Physics, 61:1040, 1974.

[20] R.E. Bruns. Mean dipole moment derivatives and anisotropies of x2cy molecules. Journal of Chemical Physics, 64:3084-3085, 1976.

[21] R.E. Bruns and R.E. Brown. The atomic mean dipole moment derivatives, anisotropies and effective charges of the diatomic hydrides. Journal of Chemical Physics, 68:880-885, 1978.

[22] W.E. Richter, L.J. Duarte, A.F. Silva, and R.E. Bruns. Review of experimental gapt and infrared atomic charges in molecules. Journal of the Brazilian Chemical Society, 27:979, 2016.

[23] J. Overend. Quantitative studies and dipole moment derivatives. In M. Davies, editor, Infrared Spectroscopy and Molecular Structure. Elsevier, 1963.

[24] B.S. Galabov and T. Dudev. Vibrational Spectra and Structure Vol 22: Vibrational Intensities. Elsevier Science, 1996.

[25] B. Galabov, T. Yamaguchi, R.B. Remington, and H.F. Schaefer III. High level ab initio quantum mechanical predictions of infrared intensities. Journal of Physical Chemistry A, 106:819-832, 2002.

[26] L.J. Duarte, A.F. Silva, W.E. Richter, and R.E. Bruns. Ftir and dispersive gas phase fundamental infrared intensities of the fluorochloromethanes: Comparison with qcisd/cc-pvtz results. Spectrochimica Acta Part A, 205:269-275, 2018.

[27] A.F. Silva, W.E. Richter, H.G.C. Meneses, and R.E. Bruns. Atomic charge transfer-counter polarization effects determine infrared ch intensities of hydrocarbons: a quantum theory of atoms in molecules model. Physical Chemistry Chemical Physics, $16: 23224,2014$.

[28] R.G. Parr, P.W. Ayers, and R.F. Nalewajski. What is an atom in a molecule? The Journal of Physical Chemistry A, 109:39573959, 2005.

[29] C.F. Matta and R.F.W. Bader. An experimentalist's reply to "what is an atom in a molecule?". The Journal of Physical Chemistry A, 110:6365-6371, 2006.

[30] Shant Shahbazian. Are there "really" atoms in molecules? Foundations of Chemistry, 16:77-84, 2014.

[31] R.F.W. Bader, A. Larouche, C. Gatti, M.T. Carroll, P.J. MacDougall, and K.B. Wiberg. Properties of atoms in molecules: Dipole moments and transferability of properties. The Journal of Chemical Physics, 87:1142, 1987.

[32] W.E. Richter, A.F. Silva, and R.E. Bruns. Atomic polarizations necessary for coherent infrared intensity modeling with theoretical calculations. The Journal of Chemical Physics, 146:134107, 2017.

[33] L.J. Saethre, M.R.F. Siggel, and T.D. Thomas. Molecular charge distribution, core-ionization energies, and the point-charge approximation. Journal of the American Chemical Society, 113:5224-5230, 1991.

[34] P.H. Guadagnini, A.E. Oliveira, R.E. Bruns, and B. Barros Neto. Core electron energies, infrared intensities and atomic charges. Journal of the American Chemical Society, 119:4224-4231, 1997.

[35] M.J. Frisch, G.W. Trucks, H.B. Schlegel, G.E. Scuseria, M.A. Robb, J.R. Cheeseman, G. Scalmani, V. Barone, B. Mennucci, G.A. Petersson, H. Nakatsuji, M. Caricato, X. Li, H.P. Hratchian, A.F. Izmaylov, J. Bloino, G. Zheng, J.L. Sonnenberg, M. Hada, M. Ehara, K. Toyota, R. Fukuda, J. Hasegawa, M. Ishida, T. Nakajima, Y. Honda, O. Kitao, H. Nakai, T. Vreven, Jr. J.A. Montgomery, J.E. Peralta, F. Ogliaro, M. Bearpark, J.J. Heyd, E. Brothers, K.N. Kudin, V.N. Staroverov, R. Kobayashi, J. Normand, K. Raghavachari, A. Rendell, J.C. Burant, S.S. Iyengar, J. Tomasi, M. Cossi, N. Rega, J.M. Millam, M. Klene, J.E. Knox, J.B. Cross, V. Bakken, C. Adamo, J. Jaramillo, R. Gomperts, R.E. Stratmann, O. Yazyev, A.J. Austin, R. Cammi, C. Pomelli, J.W. Ochterski, R.L. Martin, K. Morokuma, V.G. Zakrzewski, G. A. Voth, P. Salvador, J.J. Dannenberg, S. Dapprich, A.D. Daniels, Ö. Farkas, J.B. Foresman, J.V. Ortiz, J. Cioslowski, and D.J. Fox. Gaussian09, Revision B.01. Gaussian, Inc., Wallingford CT, 2009.

[36] T.C.F. Gomes, J.V. Silva, L.N. Vidal, P.M.A. Vazquez, and R.E. Bruns. Implementação computacional do modelo carga-fluxo de carga-fluxo de dipolo para cálculo e interpretação das intensidades do espectro infravermelho. Química Nova, 31:1750, 2008. 
342 [37] T.C.F. Gomes, J.V. Silva, L.N. Vidal, P.A.M. Vazquez, and R.E. Bruns. Chelpg and qtaim atomic charge and dipole models 343 for the infrared fundamental intensities of the fluorochloromethanes. Theoretical Chemistry Accounts, $121: 173$, 2008.

344 [38] T.A. Keith. Aimall (version 14.11.23), tk gristmill software, overland park ks, usa, 2014 (aim.tkgristmill.com).

345 [39] S.A.C. McDowell. On the negative dipole moment derivatives of hrgx ( $\mathrm{rg}=\mathrm{he}, \mathrm{ar}, \mathrm{kr} ; \mathrm{x}=\mathrm{f}, \mathrm{cl}) \mathrm{molecules}$. Journal of Molecular 346 Structure:THEOCHEM, 760:109-112, 2006.

347 [40] W.E. Richter, L.J. Duarte, A.F. Silva, and R.E. Bruns. Revisiting the negative dipole moment derivatives of hngx molecules. $348 \quad$ Theoretical Chemistry Accounts, 139:70, 2020. 